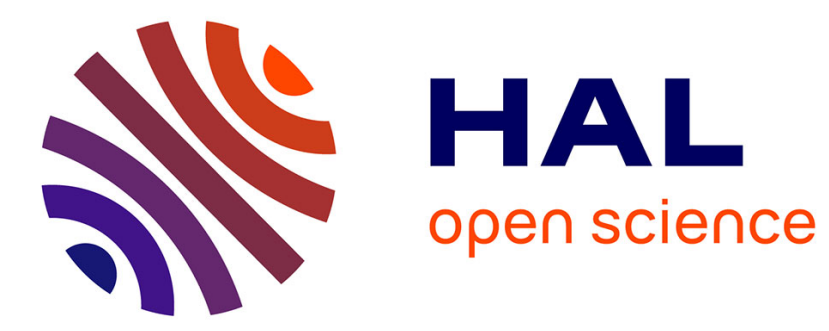

\title{
Correcting winds measured with a Rayleigh Doppler lidar from pressure and temperature effects
}

\author{
Alain Dabas, M. L. Denneulin, Pierre H. Flamant, C. Loth, Anne Garnier, A.
}

Dolfi-Bouteyre

\section{> To cite this version:}

Alain Dabas, M. L. Denneulin, Pierre H. Flamant, C. Loth, Anne Garnier, et al.. Correcting winds measured with a Rayleigh Doppler lidar from pressure and temperature effects. Tellus A, 2008, 60 (2), pp.206-215. 10.1111/j.1600-0870.2007.00284.x . hal-00245621

\section{HAL Id: hal-00245621 \\ https://hal.science/hal-00245621}

Submitted on 12 Dec 2020

HAL is a multi-disciplinary open access archive for the deposit and dissemination of scientific research documents, whether they are published or not. The documents may come from teaching and research institutions in France or abroad, or from public or private research centers.
L'archive ouverte pluridisciplinaire HAL, est destinée au dépôt et à la diffusion de documents scientifiques de niveau recherche, publiés ou non, émanant des établissements d'enseignement et de recherche français ou étrangers, des laboratoires publics ou privés. 


\section{Correcting winds measured with a Rayleigh Doppler lidar from pressure and temperature effects}

\section{A. Dabas, M. L. Denneulin, P. Flamant, C. Loth, A. Garnier \& A. Dolfi-Bouteyre}

To cite this article: A. Dabas, M. L. Denneulin, P. Flamant, C. Loth, A. Garnier \& A. Dolfi-Bouteyre (2008) Correcting winds measured with a Rayleigh Doppler lidar from pressure and temperature effects, Tellus A: Dynamic Meteorology and Oceanography, 60:2, 206-215, DOI: 10.1111/ j.1600-0870.2007.00284.x

To link to this article: https://doi.org/10.1111/j.1600-0870.2007.00284.x

\section{(c) 2007 The Author(s). Published by Taylor \& Francis.}

曲 Published online: 15 Dec 2016.

Submit your article to this journal 지

Џll Article views: 90

Q View related articles $₫$

7 Citing articles: 27 View citing articles ๘ 


\title{
Correcting winds measured with a Rayleigh Doppler lidar from pressure and temperature effects
}

\author{
By A. DABAS ${ }^{1 *}$, M. L. DENNEULIN ${ }^{1}$, P. FLAMANT ${ }^{2}$, C. LOTH ${ }^{2}$, A. GARNIER ${ }^{3}$ \\ and A. DOLFI-B OUTEYRE ${ }^{4}$, ${ }^{1}$ Météo-France, France; ${ }^{2}$ Laboratoire de Météorologie Dynamique, France; \\ ${ }^{3}$ Service d'Aéronomie, France; ${ }^{4}$ Office National de Recherches Aéronautiques, France
}

(Manuscript received 11 January 2007; in final form 23 July 2007)

\begin{abstract}
The molecular channel of the space-based Doppler lidar ADM-Aeolus relies on a double Fabry-Perot (FP) interferometer. The difference in photon numbers transmitted by the two FPs divided by their sum- the so-called Rayleigh response-is a function of the central frequency of the spectrum of the laser light backscattered by the atmosphere, so that a proper inversion enables the measurement of Doppler shifts and line-of-sight wind velocities. In this paper, it is shown that the relation-ship between the Rayleigh response and the Doppler shift does not depend on the sole characteristics of the instrument, but also on the atmospheric pressure and temperature (through the Rayleigh-Brillouin effect), and the likely presence of a narrow-band radiation due to particle scattering. The impact of these on the precision of inverted Doppler shifts (or line-of-sight winds) is assessed showing that a correction is needed. As they are lacking the appropriate precision, climatology profiles of pressure, temperature or aerosols cannot be used as an input. It is proposed to use data predicted by a numerical weather prediction system instead. A possible correction scheme is proposed. Its implication on the quality of retrieved Rayleigh winds is discussed.
\end{abstract}

\section{Introduction}

ADM-Aeolus is a space-based Doppler lidar. It will observe the wind field by analysing the frequency shift of a pulsed, laser radiation backscattered by the atmosphere. According to the Doppler effect, the frequency shift $\Delta v$ is proportional to the component $v_{\mathrm{r}}$ of the wind along the line-of-sight (LOS) of the instrument:

$\Delta v=-\frac{2 v_{\mathrm{r}}}{\lambda_{0}}$.

Here $\lambda_{0}$ is the laser wavelength, and $v_{\mathrm{r}}$ is counted positive when the wind is blowing away from the lidar. For ADM-Aeolus, $\lambda_{0}=355 \mathrm{~nm}$, so a LOS velocity $v_{\mathrm{r}}$ of $1 \mathrm{~ms}^{-1}$ causes a frequency shift $\Delta v=5.63 \mathrm{MHz}$.

There are two different types of targets that backscatter laser radiations in the atmosphere: particles (aerosols or cloud droplets) and air molecules. On the average, both types move with the wind (except for cloud droplets) so they can be used as wind tracers. However, due to thermal agitation and collisions, both also exhibit random motions. For air molecules, the dominating mechanism is the thermal agitation. The standard deviation for a single component (along the LOS for instance)

\footnotetext{
*Corresponding author.

e-mail: alain.dabas@meteo.fr

DOI: $10.1111 /$ j.1600-0870.2007.00284.x
}

of the velocity vector, is $\sigma_{v_{\mathrm{r}}}=\sqrt{\mathrm{kT} / \mathrm{m}}$, where $k=1.38 \times$ $10^{-23} \mathrm{JK}^{-1}$ is Boltzman's constant, $m=4.82 \times 10^{-26} \mathrm{~kg}$ is the mass of a single air molecule and $\mathrm{T}$ is the temperature. It results in a broadening of the spectrum of the backscattered light, which width is then given by

$\sigma_{\Delta v}=\sqrt{\sigma_{\text {las }}^{2}+4 \frac{\sigma_{v_{\mathrm{r}}}^{2}}{\lambda_{0}^{2}}} \approx \frac{2}{\lambda_{0}} \sqrt{\frac{\mathrm{kT}}{\mathrm{m}}}$,

where $\sigma_{\text {las }} \approx 33 \mathrm{MHz}$ is the spectral width of the emitted laser pulse (assuming a Fourier-transform-limited spectrum of the $\sim 30 \mathrm{~ns}$ pulse). Note that the right-hand side term is a good approximation because $\sigma_{\text {las }} \ll 2 \sigma_{v_{\mathrm{r}}} / \lambda_{0}$. Indeed, for $T=300 \mathrm{~K}$ $\sigma_{v_{\mathrm{r}}} \approx 293 \mathrm{~ms}^{-1}$ and $\sigma_{\Delta v}=2 \sigma_{v_{\mathrm{r}}} / \lambda_{0}=1.65 \mathrm{GHz}$.

As far as aerosol particles or cloud droplets are concerned, their mass (a $1 \mu \mathrm{m}$-size droplet made of water weights $4.2 \times$ $10^{-15} \mathrm{~kg}$ ) combined with collision effects with air molecules results into a LOS wind dispersion of the order of $1 \mathrm{~mm} \mathrm{~s}^{-1}$ or less. The spectral broadening effect on the return signal is thus negligible; the spectrum of the backscattered light is almost equal to the spectrum of the emitted laser pulse.

ADM-Aeolus was designed for measuring winds from both particles and molecules. Particle backscatter is used wherever particles are present in sufficient concentrations, it offers the advantage of narrow return spectra to the benefit of a better precision for wind measurements. Molecules are used where 
particles are otherwise scarce-at high altitudes for instance. For ADM, a noticeable advantage is the capacity to make wind measurements up to altitudes of 25 or $30 \mathrm{~km}$, that is in the upper troposphere/lower stratosphere (UTLS), thus documenting vertical exchanges at the global scale in a sensitive interface region of the atmosphere.

Since the molecular and particle spectra have totally different widths, their simultaneous detection within a single instrument requires the implementation of two separate detection channels, one designed for large spectra, the other one for narrow ones.

In ADM, the narrow-spectrum detection channel-the socalled Mie channel-is based on a fringe imaging technique. Such a technique was proposed by several authors in the past, see, for example, Killeen et al. (1983), Abreu et al. (1992) or McGill et al. (1997), but the type of interferometer used by ADM-a Fizeau interferometer-was never used before for a Doppler lidar application.

As far as the large-spectrum detection channel is concernedthe so-called Rayleigh channel-the solution retained for ADM is inspired by the pioneering developments conducted in France in the late $80 \mathrm{~s}$ and early $90 \mathrm{~s}$ by M. L. Chanin and her team (see Chanin et al., 1989, Garnier and Chanin, 1992, Souprayen et al., 1999a and b) and implemented at the Observatoire de Haute Provence (OHP). The detection channel is composed of a pair of Fabry-Perot (FP) interferometers with centre frequencies located on either side of the emitted laser frequency (see details in Section 1 below) so that the number of photons transmitted by the two FPs are nearly equal in the absence of wind (see Fig. 1). When the wind is blowing the return spectrum is Doppler shifted (see dashed spectrum in Fig. 1), and the number of photons from one FP $\left(N_{\mathrm{A}}\right.$ in Fig. 1) increases while it decreases at the output of the other one $\left(N_{\mathrm{B}}\right.$ in Fig. 1). The difference in photon numbers $N_{\mathrm{A}}-N_{\mathrm{B}}$ thus bear a signature of the Doppler shift which, by a proper calibration and inversion mechanism, can be used for measuring LOS winds.

However, the relationship between the photon number difference and the LOS wind depends on the precise shape of the return spectrum, which is in turn dependent on atmospheric parameters. In Garnier et al. (1992), two factors are identified, the temperature and the presence of Mie backscatter in the return spectrum. The temperature modifies the width of the molecular return (see eq. 2) and Mie backscatter adds a narrow peak on top of the broad Rayleigh spectrum. For the wind lidar operating at OHP, the temperature effect is corrected on the basis of temperature profiles provided by a radio-sonde launched nearby or from a model such as European Centre for Medium Range Weather Forecast (ECMWF). As far as Mie backscatter is concerned, some information can be obtained from the strength of the Rayleigh return, it can be used to tag the Rayleigh measurements presumably contaminated by Mie return, but is not accurate enough to correct for it.

For ADM, taking into account the temperature and Mie contamination is a necessity, but the approach followed at OHP is

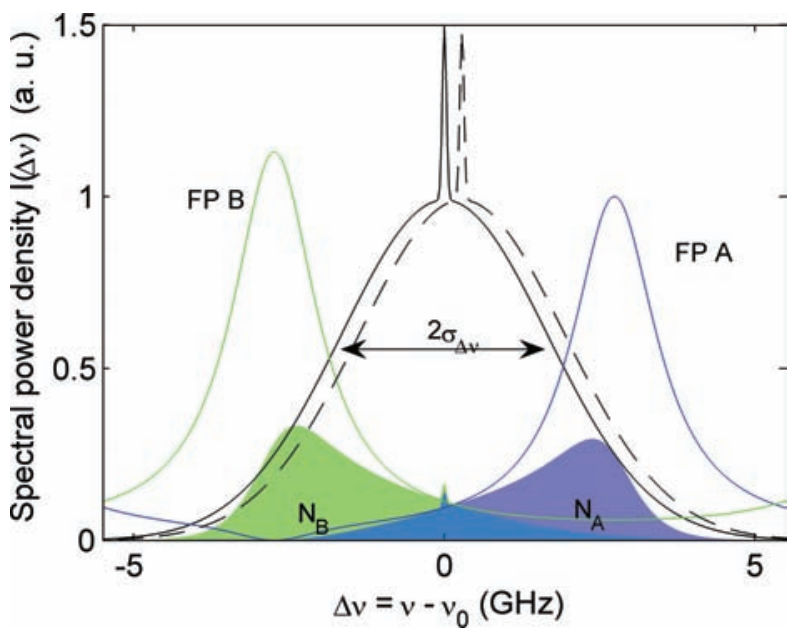

Fig. 1. Diagram showing how the double-FP receiver of the Rayleigh channel of ADM-Aeolus is operating. A typical spectrum of atmospheric backscatter is displayed with a black, solid line. The broad par of half $-1 / e^{2}$-width is caused by the air molecules, the narrow peak on its top is due to backscattering particles. The spectrum is centred about the frequency $v_{0}$ of the emitted laser pulse. The transmission functions of FP A (right-hand side) and B (left-hand side) are shown with a blue and a green solid line. They are representative of the nominal ADM Rayleigh receiver. The blue and green shades are obtained by multiplying the spectrum by the transmission function of FP A (blue) and B (green), their area is proportional to the numbers $N_{\mathrm{A}}$ and $N_{\mathrm{B}}$ of photons transmitted by the two FPs. If the spectrum is Doppler shifted (see the black, dashed line) to a positive centre frequency (here $282 \mathrm{MHz}$, or $50 \mathrm{~ms}^{-1}$ in terms of LOS velocity), the number $N_{\mathrm{A}}$ increases while $N_{\mathrm{B}}$ decreases. The total number of photons $N_{\mathrm{A}}+N_{\mathrm{B}}$ remains nearly constant, so the Rayleigh response $R_{\mathrm{R}}=\left(N_{\mathrm{A}}\right.$ $\left.-N_{\mathrm{B}}\right) /\left(N_{\mathrm{A}}+N_{\mathrm{B}}\right)$ increases.

not applicable because a preliminary study conducted at the European Space Agency (ESA) showed that pressure-dependant Brillouin scattering may introduce significant errors in Rayleigh winds.

As a result, ESA initiated a study on the potential impact on ADM Rayleigh winds of pressure, temperature and Mie contamination. The objectives were three-fold: (1) review the existing literature available on Rayleigh-Brillouin scattering and assess the relevance of published theories for accurately predicting spectra of light backscattered by air molecules, (2) assess the impact of temperature, pressure and Mie contamination on Rayleigh winds and (3) propose correction schemes whenever required. The final report (Flamant et al., 2005) can be obtained from the European Space Agency.

The present paper presents the results obtained on objectives 2 and 3. The biasing impact of pressure, temperature and Mie contamination on Rayleigh winds is quantified, it is shown that although moderate, it exceeds the requirements on absolute errors set for the mission. A correction scheme is proposed, it will be implemented in the level $2 \mathrm{~b}$ processor at ECMWF or any 
meteorological service that intends to assimilate ADM data. A full description of the level 2 processor is given in a companion paper (Tan et al., 2007). As part of the data assimilation process of a numerical weather prediction system, it uses temperature and pressure fields predicted by the NWP model at the previous run as input.

The paper is organized in five sections. Section 2 presents the Rayleigh detection channel of ADM, its major components, how it works, and clarifies why it is affected by pressure, temperature and Mie contamination. In Section 3, the impact of these effects is quantified. In Section 4, a scheme-the so-called RayleighBrillouin Correction (RBC) scheme is proposed for correcting pressure and temperature effects. Section 5 shows how Mie contamination can be treated. At last, the conclusion summarizes the previous sections and rehash the impact the RBC scheme may have on winds assimilated by the NWP systems.

\section{ADM-Aeolus Rayleigh receiver}

The Rayleigh channel of ADM-Aeolus mostly consists of a double FP interferometer and a CCD. It works as follows. The light backscattered by the atmosphere is transmitted through the two FPs. The number of photons $N_{\mathrm{A}}$ and $N_{\mathrm{B}}$ in the two channels are counted by a CCD unit. Then the so-called Rayleigh $R_{\mathrm{R}}$ response is computed

$R_{\mathrm{R}}=\frac{N_{\mathrm{A}}-N_{\mathrm{B}}}{N_{\mathrm{A}}+N_{\mathrm{B}}}$.

The relationship between the spectrum backscattered by the atmosphere and the characteristics of the double FP is illustrated in Fig. 1. A typical backscattered spectrum is displayed with a black, solid line. It is made of a broad spectrum representative of the molecular backscatter (its half- $1 / e^{2}$ width is given by eq. 2 ), and a narrow peak due to particles. In the figure, the relative contribution of Mie scattering is $1 \%$ of the total backscatter. It results in a scattering ratio $\rho=1+\beta_{\text {aer }} / \beta_{\text {mol }}=1.01$ where $\beta_{\text {aer }}$ and $\beta_{\text {mol }}$ are the particle and molecular backscatter ratios of the atmospheric volume under consideration. The filtering characteristics of FPs A and B are shown with a blue and a green solid line (representative of the nominal dual FP receiver of ADM). The amount of the initial spectrum transmitted by the FPs is represented by the shaded areas obtained by multiplying the input spectrum by the FP transmission curves. It can easily be seen that a Doppler shifting of the spectrum (the black dashed line shows the input spectrum shifted to a positive frequency of $262 \mathrm{MHz}$ equivalent to a $50 \mathrm{~ms}^{-1} \mathrm{LOS}$ wind), results in $N_{\mathrm{A}}>N_{\mathrm{B}}$, while $N_{\mathrm{A}}+N_{\mathrm{B}}$ varies only slightly, so the response $R_{\mathrm{R}}$ increases.

The retrieval of the Doppler shift or equivalent LOS wind speed is made by inverting the relationship between $R_{\mathrm{R}}$ and the centre frequency $v_{d}$ of the Doppler shifted spectrum. Let us denote $T_{\mathrm{A}}(v)$ and $T_{\mathrm{B}}(v)$ the transmission curves of FP A and B, and $\mathrm{I}\left(v-v_{\mathrm{d}}\right)$ the return spectrum. The numbers $N_{\mathrm{A}}$ and $N_{\mathrm{B}}$ are then given by

$N_{\mathrm{A}, \mathrm{B}}\left(v_{\mathrm{d}}\right)=K \int_{-\mathrm{FSR} / 2}^{+\mathrm{FSR} / 2} T_{\mathrm{A}, \mathrm{B}}(v) I\left(v-v_{\mathrm{d}}\right) \mathrm{d} v$,

where FSR is the free spectral range of the FPs to which the CCD is matched and $K$ is a calibration constant valid for FPs $\mathrm{A}$ and $\mathrm{B}$ which precise value does not need to be known ( $K$ cancels out when $R_{\mathrm{R}}$ is computed). It is clear from eq. (4) that the relationship between $R_{\mathrm{R}}$ and $v_{\mathrm{d}}$ depends in a complex manner on the FP characteristics through the functions $T_{\mathrm{A}}$ and $T_{\mathrm{B}}$, but also on the atmosphere through the shape of $I(v)$.

A good model for $I(v)$ is

$I(v)=I_{\mathrm{RB}}(v)+(\rho-1) I_{\mathrm{mie}}(v)$,

where $\rho$ is the scattering ratio (ratio of the particle backscatter $\beta_{\text {part }}$ to the sum of the particle and molecular backscatters $\left.\beta_{\text {part }}+\beta_{\text {mol }}\right), I_{\text {mie }}(v)$ is the narrow spectrum due to the particles (the narrow peak in Fig. 1), and $I_{\mathrm{RB}}(\nu)$ is the broad, molecular spectrum. Here, the subscript RB stands for Rayleigh-Brillouin.

Usually, $I_{\mathrm{RB}}(v)$ is modelled by a Gaussian spectrum with a width given by eq. (2). However, theoretical and experimental works (Bathnagar et al., 1954; Yip and Nelkin, 1964; Boley et al., 1972; Tenti et al., 1974; Clark, 1975; Wegdam and Schaink, 1989; Rye, 1998) have shown it is only an approximation valid in the so-called Knudsen regime where the mean-free path of the scatters, that is, the average distance they are travelling between two successive collisions in the gas medium, is much longer than the probing laser wavelength.

In the kinetic regime, the ratio of the laser wavelength to the mean-free path can be approximated in the atmosphere by

$y=0.23 P(\operatorname{atm}) \lambda_{0}(\mathrm{~nm}) \frac{T(K)+111}{T^{2}(K)}$

which is used in the Tenti S6 model (see Pan et al., 2002b). Figure 2 shows the value of the y parameter for a typical atmosphere (US Standard 76) and ADM laser wavelength $\lambda_{0}=355$ $\mathrm{nm}$. It appears that the condition $y \ll 1$ required for the validity of the classical Knudsen regime is met only at high altitudes. Below, $y$ is of the order of 1 , that is, in the so-called kinetic regime. In this regime, the theory predicts the emergence of two 'Brillouin' peaks — or 'doublets' —on either side of the Gaussian spectrum. The peaks are centred about the frequencies $\pm \sigma_{\Delta v}$, and their magnitude is a function of $y$. They disappear when $y \rightarrow 0$. The shape of the resulting Rayleigh-Brillouin spectrum [hence the RB subscript in $I_{\mathrm{RB}}(v)$ ] is displayed for typical surface conditions $(P=1013 \mathrm{hPa}$ and $T=288 \mathrm{~K})$ in Fig. 3 . The RB spectrum was computed by using the Tenti S6 model (see Tenti et al., 1974, Pan et al., 2002a and b, 2004) that is widely used today as a diagnostic tool for combustion physics and supersonic flows, despite its inherent deficiencies due to the fact that it implements various parameters valid for $\mathrm{N}_{2}$ molecules and not air. The comparison with the standard, Gaussian spectrum of width $\sigma_{\Delta v}$ shows a small, but significant departure. We will see in the 


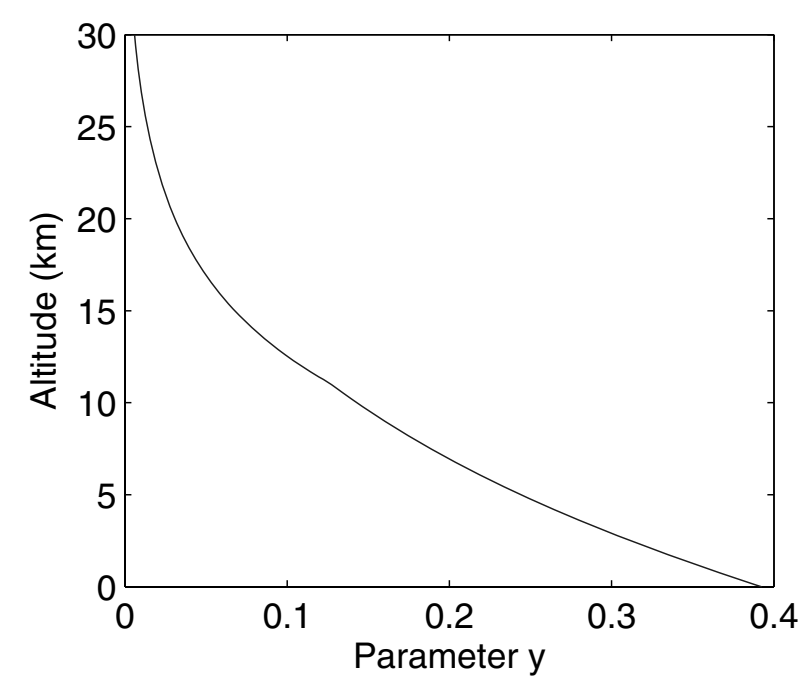

Fig. 2. Parameter $y$ (eq. 3) for pressure and temperature profiles from the US 76 Standard atmosphere and a wavelength $\lambda=355 \mathrm{~nm}$. It can be seen $y$ is of the order of 1 up to high altitudes above $10 \mathrm{~km}$, so a significant pressure impact (Brillouin doublets) is expected for ADM.

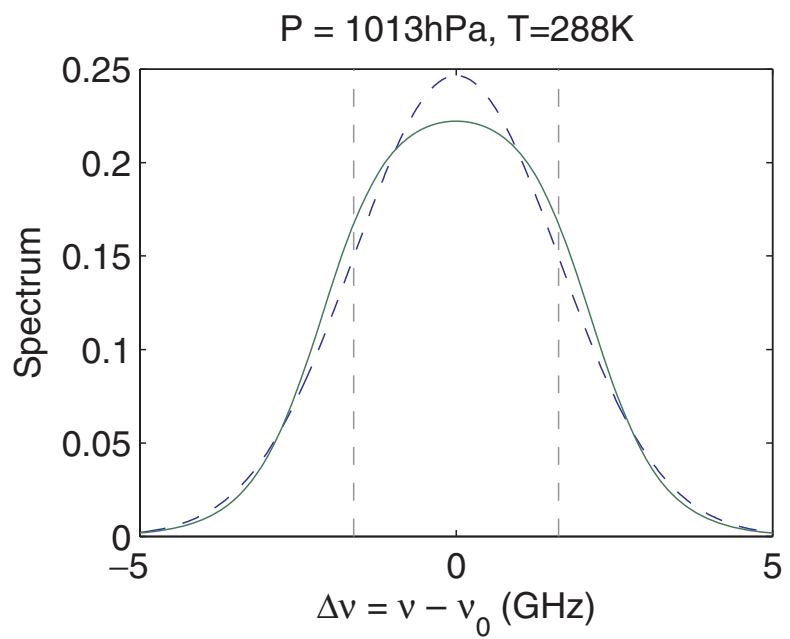

Fig. 3. Rayleigh-Brilllouin (green solid line) and Rayleigh (dashed, blue) spectra for a pressure $P=1013 \mathrm{hPa}$ and a temperature $T=288 \mathrm{~K}$ corresponding to the conditions at ground of the US Standard 76 atmosphere (parameter $y$ is $\sim 0.4$ ). The dashed, vertical bars are located at $\Delta v= \pm \sigma_{\Delta v}$ where the Brillouin doublets are centred.

next section that it has a significant impact on the response of the Rayleigh receiver of ADM and thus it must be taken into account.

In this paper, we use Tenti S6 model for assessing the impact of pressure and temperature on Rayleigh wind retrievals although it has not been validated for air where in addition phase changes may occur. We assume that the model does give at least a good indication on the potential impact of Brillouin scattering in the atmosphere so that the conclusions drawn in the next section on the necessity to correct Rayleigh winds from pressure and tem- perature are valid. As far as the RB correction scheme proposed in Section 3 is concerned, it must be outlined that it needs a RB model, but what precise model does not matter. If a new, better proven model arises (laboratory experiments are currently being conducted), the correction scheme would still be working, but the tables it is based upon would change.

\section{Impact of temperature, pressure and Mie contamination on Rayleigh wind retrieval}

\subsection{Mission requirements}

In the subsections below, the impact of pressure, temperature and Mie contamination on Rayleigh winds is quantified and the need for a correction is assessed. The assessment is made relative to ADM-Aeolus mission requirements on wind retrieval accuracy and precision. These requirements are summarized here.

The accuracy - that is the absolute value of the difference between the statistical mean of radial wind measurement and the true radial wind $v_{\mathrm{r}}$-must not exceed $0.23 \mathrm{~ms}^{-1}+0.007 \times\left|v_{\mathrm{r}}\right|$ where $\|$ denotes the absolute value. The major sources of systematic errors in ADM are the calibration errors, the uncertainty of the satellite velocity and attitude, the incidence angle of the light wave returned by the atmospheric relative to the axis of the FPs... The current design of the lidar instrument and its operating modes (calibration modes in particular) is such that the actual accuracy is close to the maximum allowed by the specification.

The precision - that is the standard deviation of measured radial velocities about their statistical mean-must be less than $1.2 \mathrm{~ms}^{-1}$ between the earth surface and the altitude of $2 \mathrm{~km}$, $1.8 \mathrm{~ms}^{-1}$, between 2 and $16 \mathrm{~km}$ and $3 \mathrm{~ms}^{-1}$ above. The major source of random errors is the photo-counting process that follows a Poisson's statistics. Example of precision levels predicted for ADM in typical atmospheric conditions can be found in the companion paper by Tan et al. They are typically comprised between 0.5 and 1 time the specification.

As we will see below, the pressure, temperature and Mie contamination must be considered as sources of systematic errors because they modify the sensitivity curve of the instrument (relationship between the radial wind $v_{\mathrm{r}}$ and the response $R_{\mathrm{R}}$ ). This is why their impact is compared to the $0.7 \%$ slope error of the accuracy specification (the 0.007 coefficient in the equation above that defines the maximum value for systematic errors).

\subsection{Temperature}

The sensitivity of the Rayleigh response with respect to the temperature inside the sensing volume is illustrated in Fig. 4. There the response curves (Rayleigh response versus temperature) are drawn for temperatures ranging from 250 to $300 \mathrm{~K}$. The responses were obtained by using eq. (3) and (4) and setting the return spectrum $I(v)$ to $I_{\mathrm{RB}}(v)$ (that is, $\rho=1$, there is no contamination of the Rayleigh light by Mie backscatter). 


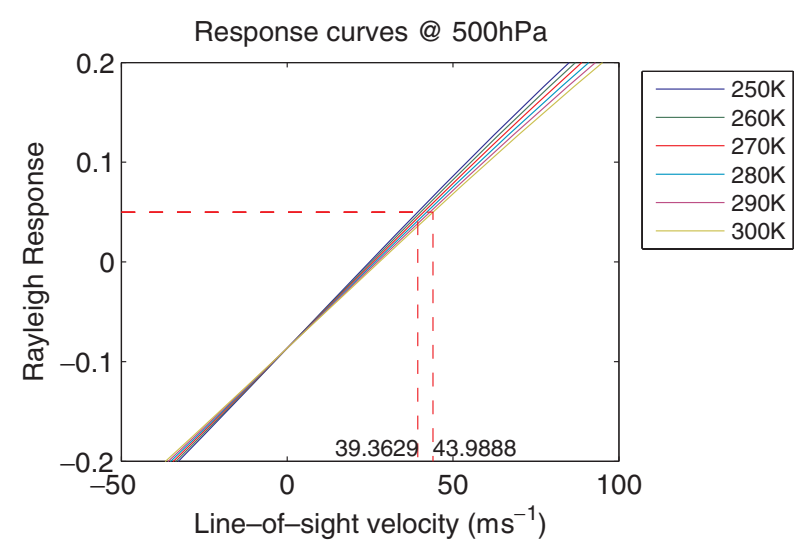

Fig. 4. Theoretical response curves of ADM lidar with respect to the temperature inside the sensing volume and a pressure of $500 \mathrm{hPa}$ (about $5 \mathrm{~km}$ altitude). The red, dashed lines show the impact of the temperature on the inversion of a Rayleigh response $R_{\mathrm{R}}=0.05$. The LOS wind varies from 39.36 to $43.99 \mathrm{~ms}^{-1}$ when the temperature increases from 250 to $300 \mathrm{~K}$. This shows an error of several meters per second is likely to be made on the LOS wind if a reference temperature, or a reference temperature profile, is considered for the inversion of Rayleigh responses. Moreover, the errors that would hence be made are tightly correlated to the actual temperature field. This kind of correlation must be avoided, they may generate physically meaningful but unrealistic dynamic features in the atmospheric fields analysed by NWP models.

$I_{\mathrm{RB}}(v)$ is computed with the Tenti S6 model for which we developed a code in MATLAB on the basis of the equations in Pan et al. (2002a). The notation $\left.I_{\mathrm{RB}}(v)\right|_{P, T}$ outlines that the model is pressure and temperature dependant. The spectra were normalized so that $\left.\int I_{\mathrm{RB}}(v)\right|_{P, T} \mathrm{~d} v=1$ whatever the pressure $P$ and the temperature $T$. Figure 4 shows that the so-called calibration curves - that is, the curves relating the Doppler shift $v_{d}$ to the Rayleigh response $R_{\mathrm{R}}$-are almost linear, all crossing at the same point, but with slightly different slopes. The impact on LOS wind retrieval is illustrated by the red, dashed lines. The Rayleigh response $R_{\mathrm{R}}=0.05$ (horizontal line) is inverted. For a temperature of $250 \mathrm{~K}$, the inverted LOS wind is $v_{\mathrm{r}}=39.36 \mathrm{~ms}^{-1}$, for $T=$ $300 \mathrm{~K}$, it is $v_{\mathrm{r}}=43.99 \mathrm{~ms}^{-1}$. The $50 \mathrm{~K}$ temperature interval thus results in an uncertainty on the LOS wind retrieval of $4.33 \mathrm{~ms}^{-1}$ or $10 \%$ of the true LOS wind speed. This is to be compared to the maximum wind slope error of $0.7 \%$ allowed for the mission. As confirmed by Fig. 5, where $\partial v_{r} / \partial T$ is plotted as a function of $\mathrm{T}$ and pressure $\mathrm{P}$ (or altitude), a good rule of thumb for ADM is a $1 \mathrm{~K}$ error on the actual temperature inside the sensing volume leads to a relative error of $0.2 \%$ of the true LOS wind. Compared to the maximum slope error of $0.7 \%$, it shows that the temperature must be known with an uncertainty less than a few K. This is obviously beyond the reach of any climatology but is in line with the accuracy achieved by NWP models. This explains why the Rayleigh winds produced by the level $1 \mathrm{~B}$ processor of ADM cannot reach the quality required by NWP users (the level 1B

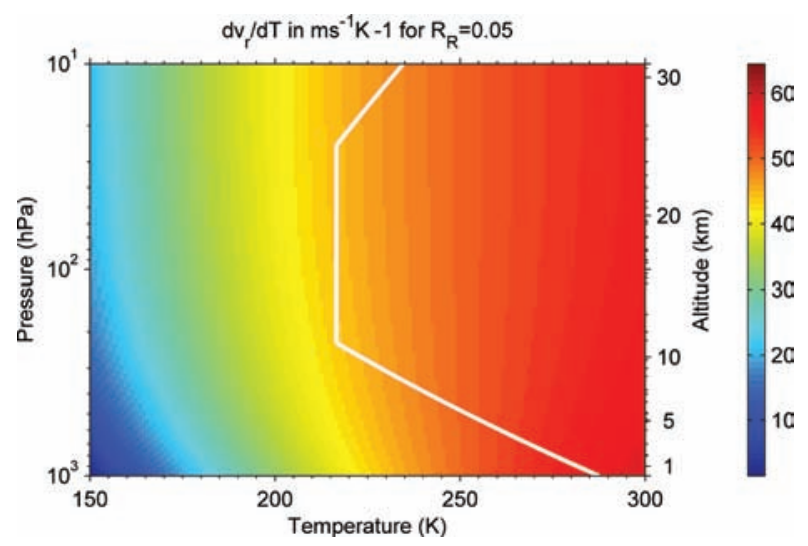

Fig. 5. First order derivative of the inverted Rayleigh LOS wind with respect to the temperature $T$ as a function of $T$ ( $x$-axis) and pressure (left $y$-axis) or altitude (right $y$-axis) for a Rayleigh response $R_{\mathrm{R}}=$ 0.05 . The thick, white, solid line is a standard temperature profile from the US 76 model. It can be seen that an error of $1 \mathrm{~K}$ on the temperature will generate an error of the order of $10 \mathrm{~cm} \mathrm{~s}^{-1}$ on the LOS wind (or $0.25 \%$ of the true wind).

processor has no access to 'external' data) and must be refined before they are actually assimilated. Given the level of accuracy needed for the temperature, the refinement must be done locally at the NWP centre by an appropriate code run just before or within the assimilation cycle. This constraint was one of the reasons why a level $2 \mathrm{~B}$ processor had to be developed. We refer to Tan et al. (2007) for details on that processor.

\subsection{Pressure}

The necessity to take into account the broadening impact of pressure on molecular return spectra (in other words, the Brillouin effect) is illustrated by Fig. 6. There are plotted with colour codes

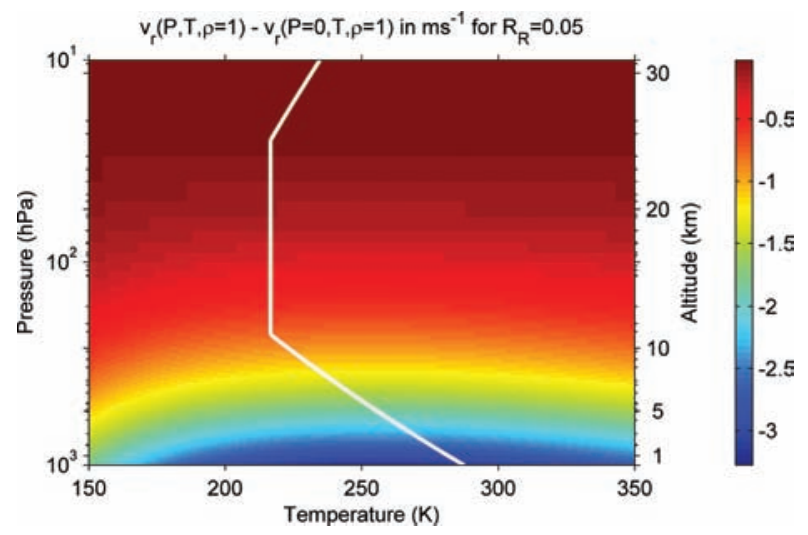

Fig. 6. LOS wind error made when pressure (Brillouin) effect on molecular return spectrum is not taken into consideration. The thick, white, solid line is a standard temperature profile from the US 76 model. The figure shows that Brillouin effect may generate errors of several meters per second on the LOS wind. 
the differences in LOS wind retrievals when the pressure effect is considered $\left[v_{r}(P, T, \rho=1)\right]$ and when it is not $\left[v_{r}(P=0\right.$, $T, \rho=1)]$. For both options, a reference response $R_{\mathrm{R}}=0.05$ was considered, corresponding to a LOS wind of about $40 \mathrm{~ms}^{-1}$ (see Fig. 4), and the scattering ratio was set to $\rho=1$, meaning that there is no contamination of molecular spectra by Mie light. As previously, $v_{r}(P, T, \rho=1)$ was computed by numerically inverting the response curves $v_{d} \rightarrow R_{\mathrm{R}}$ for the pressure $P$ and temperature $T$ under consideration. The response curves were obtained by a numerical integration of eq. (4) by a finite difference scheme with a frequency step $\mathrm{d} f=25 \mathrm{MHz}$. For the return spectra, we used Tenti S6 model normalized to a unit power $\left(\int I_{\mathrm{RB}}(v) \mathrm{d} v=1\right)$. We recall that this model converges to the Gaussian power density

$\left.I_{\mathrm{RB}}(v)\right|_{P=0, T}=\frac{1}{\sqrt{2 \pi} \sigma_{\Delta v}} \exp \left(-\frac{v^{2}}{2 \sigma_{\Delta v}^{2}}\right)$,

where $\sigma_{\Delta v}$ is given by eq. (2) when the pressure $P$ tends to 0 (no Brillouin effect).

Figure 6 shows that the Brillouin effect may generate LOS wind retrieval errors of up to several meters per second. As expected, the errors are growing with the pressure, they reach their largest magnitude at the surface. At the altitude of $30 \mathrm{~km}$ - the highest altitude that ADM can usefully probe-the errors are still of the order of a few tens of centimetres per second. This is not much, but still highlights the necessity for a proper correction.

Figure 7 gives an indication on what accuracy is needed on the pressure for correcting the Brillouin effect. The figures gives the first-order derivative of $v_{\mathrm{r}}$ with respect to the pressure $P$, as a function of the temperature ( $x$-axis) and the pressure ( $y$-axis). For typical atmospheric conditions (see white, thick line), $\partial v_{r} / \partial P \approx$ $0.003 \mathrm{~ms}^{-1}(\mathrm{hPa})^{-1}$. The maximum wind slope error is still met with a pressure uncertainty of $\sim 100 \mathrm{hPa}$ (the error on $v_{\mathrm{r}}$ is then equal to $\sim 30 \mathrm{~cm} \mathrm{~s}^{-1}$ or $0.7 \%$ of the true radial wind), so the use of

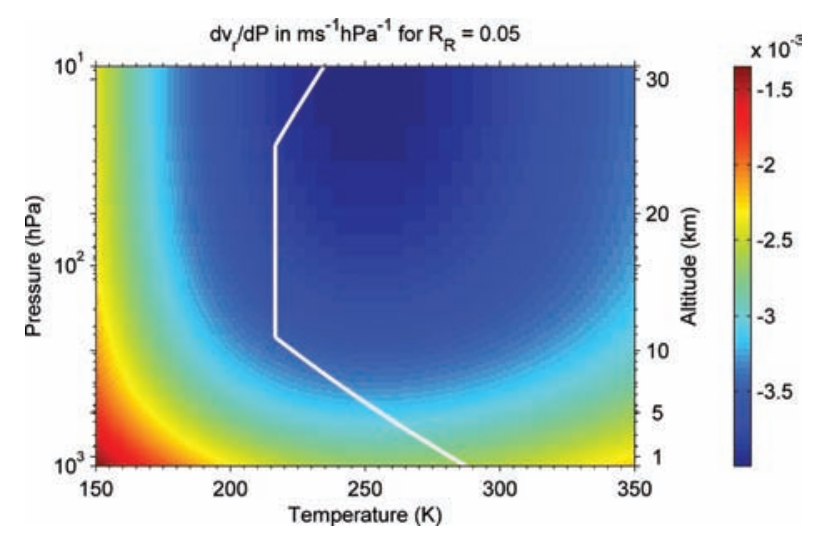

Fig. 7. First order derivative of the inverted Rayleigh LOS wind with respect to the temperature $T$ as a function of $T$ ( $x$-axis) and pressure (left $y$-axis) or altitude (right $y$-axis) for a Rayleigh response $R_{\mathrm{R}}=$ 0.05 . The thick, white, solid line is a standard temperature profile from the US 76 model.

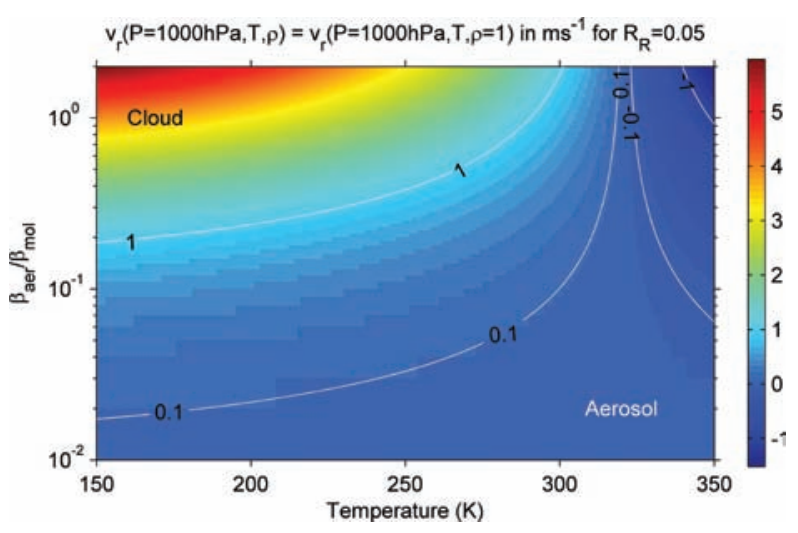

Fig. 8. LOS wind errors generated by Mie contamination at the surface $(P=1000 \mathrm{hPa})$, as a function of the temperature ( $x$-axis), and aerosol to molecular backscatter ratio $\beta_{\text {aer }} / \beta_{\text {mol }}$. The true LOS wind is of the order of $40 \mathrm{~ms}^{-1}\left(R_{\mathrm{R}}=0.05\right)$. Due to the short UV wavelength of ADM, the ratio $\beta_{\text {aer }} / \beta_{\text {mol }}$ does rarely exceed a few percents in aerosol layers with associated LOS wind errors less than $0.1 \mathrm{~ms}^{-1}$.

climatology profiles for $P$ is possible in principle. However, the NWP model output required for correcting temperature effects on Rayleigh winds predicts the pressure with uncertainty levels of less than a few $\mathrm{hPa}$, thus enabling the correction of pressure effects to an accuracy of a few centimetres per second which is negligible compared to the maximum accuracy required by mission specifications.

\subsection{Mie contamination}

The impact of Mie contamination of Rayleigh winds is illustrated in Fig. 8 where the first-order derivative $\partial v_{r} / \partial \rho$ is displayed as a function of the temperature $T$ and the relative strength of the aerosol contribution characterized by $\rho-1=\beta_{\text {aer }} / \beta_{\text {mol }}$. Due to the short, UV wavelength of $\operatorname{ADM}\left(\beta_{\text {mol }}\right.$ scales with $\lambda_{0}^{-4}$ while $\beta_{\text {aer }}$ follows a power law $\lambda_{0}^{-\alpha}$ with $\alpha \approx 1$ ), typical values for $\beta_{\text {aer }} / \beta_{\text {mol }}$ are of the order of a few hundreds in aerosol layers in the atmosphere, with larger values to be found only in aerosol layers of particularly thick optical depth or in clouds. There it can reach several tens or even several units. Having this in mind, it turns out that LOS wind error should not exceed a few thousands (errors less than $0.1 \mathrm{~ms}^{-1}$ for a true wind $\sim 40 \mathrm{~ms}^{-1}$ ) of the true wind speed in most cases, which is within the requirement set for $R_{\mathrm{R}} \rightarrow v_{\mathrm{r}}$ slope errors $(0.7 \%)$. From this, it could be concluded that a correction for Mie contamination is not necessary, because: (1) for common values of $\beta_{\mathrm{aer}} / \beta_{\mathrm{mol}}$, the error on the retrieved LOS wind is much less than the required accuracy, (2) for large values of $\beta_{\mathrm{arr}} / \beta_{\mathrm{mol}}$ where wind errors exceed the requirement, the quality of the signal from the Fizeau should allow a good measurement on the Mie channel of the lidar. In that perspective, what is needed is a reasonably good estimator of $\beta_{\text {aer }} / \beta_{\text {mol }}$ in order to flag out Rayleigh LOS winds and validate Mie LOS wind when $\beta_{\text {aer }} / \beta_{\text {mol }}$ exceeds a predefined threshold, 
and conversely validate the Rayleigh LOS wind and flag out the Mie LOS wind when $\beta_{\mathrm{aer}} / \beta_{\mathrm{mol}}$ is below another threshold. Nevertheless, a correction scheme was developed for Mie contamination. Explained in Section 5, it comes as a small addition to the pressure-temperature correction carried out by the level $2 \mathrm{~B}$ processor according to the algorithm presented in Section 4 below. Its impact on the quality of ADM measurements may turn out to be small, but it should improve the quality of the winds in the regions of intermediate $\beta_{\text {aer }} / \beta_{\text {mol }}$ where Mie contamination may become significant for Rayleigh winds but not high enough to guarantee a precise Mie measurement. The application of the Mie-decontaminator requires an input value for $\rho$. It cannot be obtained from the NWP model, neither directly (there is currently no $\beta_{\text {aer }}$ variable in NWP models) nor indirectly (there does not exist any useful relationship between model parameters and $\beta_{\text {aer }}$ ), so it has to come from another source. The precision required for it - of the order of a few hundreds as shown by simulations not presented here-rules out the use of a climatology. The only remaining option is thus an estimation from ADM signals. Such an estimation is possible in the case the atmospheric volume sensed by the Rayleigh receiver is also probed by the Mie channel. Then the analysis of the Mie return spectrum provides the required information. We do not detail the algorithm for it here, but it is based on the estimation of the relative levels of the useful-peaked and noisy-flat parts of the spectrum imaged at the output of the Fizeau. In case there is no information available from the Mie channel (this may happen for instance at the highest altitudes where the Mie detector is no more sensitive under the nominal settings of the instrument), then no value for $\rho$ is available and the Mie-decontaminator lacks the input it needs to operate. In the level 2B processor, this type of situation is detected, the Mie-decontamination stage is by-passed, a Rayleigh LOS wind corrected from temperature and pressure effects is output, but is flagged consequently.

\section{Rayleigh-Brillouin correction scheme}

In this section, we assume that Rayleigh photocounts $N_{\mathrm{A}}$ and $N_{\text {B }}$ suffer no contamination by Mie backscatter. In that case, the retrieval of a Doppler shift (or a LOS wind) from an observed Rayleigh response $\hat{R}_{\mathrm{R}}$ consists in inverting eq. (3) with $N_{\mathrm{A}}$ and $N_{\mathrm{B}}$ given by eq. (4) and $I(v)=\left.I_{\mathrm{RB}}(v)\right|_{P, T}$ provided by an appropriate model. In this study, we used the Tenti S6 model. The inversion can be done by a simple numerical scheme. Given the pressure $P$ and $T,\left.I_{\mathrm{RB}}(v)\right|_{P, T}$ is computed for a large and wide array of frequencies, it is convolved with the transmission curves $T_{\mathrm{A}}(v)$ and $T_{\mathrm{B}}(v)$ characterized by a proper calibration in order to obtain $N_{\mathrm{A}}$ and $N_{\mathrm{B}}$ for a large array of frequency Doppler shifts $v_{d}$ paving the Useful Spectral Range (USR) of the receiver (USR $=[-750 \mathrm{MHz},+750 \mathrm{MHz}]$ by instrument design), and then a simple interpolation scheme (linear for instance) is used in order to determine which Doppler shift produces the response $\hat{R}_{\mathrm{R}}$. The whole process is summarized in Fig. 9. Numerical tests not reported here showed that a precise inversion of Rayleigh responses - that is, inversion with residual errors less than a few millimetres per second-is achieved provided the algorithm is carefully implemented. For instance, the frequency step of the finite difference used for approximating the convolution product of eq. (4) must of the order of $\mathrm{d} f=25 \mathrm{MHz}$ or finer. With a frequency step of $50 \mathrm{MHz}$ for instance, residual errors grow up to a few centimetres per second, which become notable.

From a computational point of view, the Tenti S6 model and the convolution product in eq. (3) are both expensive since they have to be repeated many times and involve large frequency arrays. (nearly 1000 samples for $T_{\mathrm{A}, \mathrm{B}}()$ and $I()$ in eq. (3) with a $\mathrm{FSR}=10950 \mathrm{MHz}$ and a d $f=25 \mathrm{MHz}$ ). To shorten the computation time of the level 2B processor of ADM, it was decided to implement the inversion process above through a look-up-table approach. In this approach, a big $3 \mathrm{D}$ matrix $\Phi(i, j, k)$ is computed

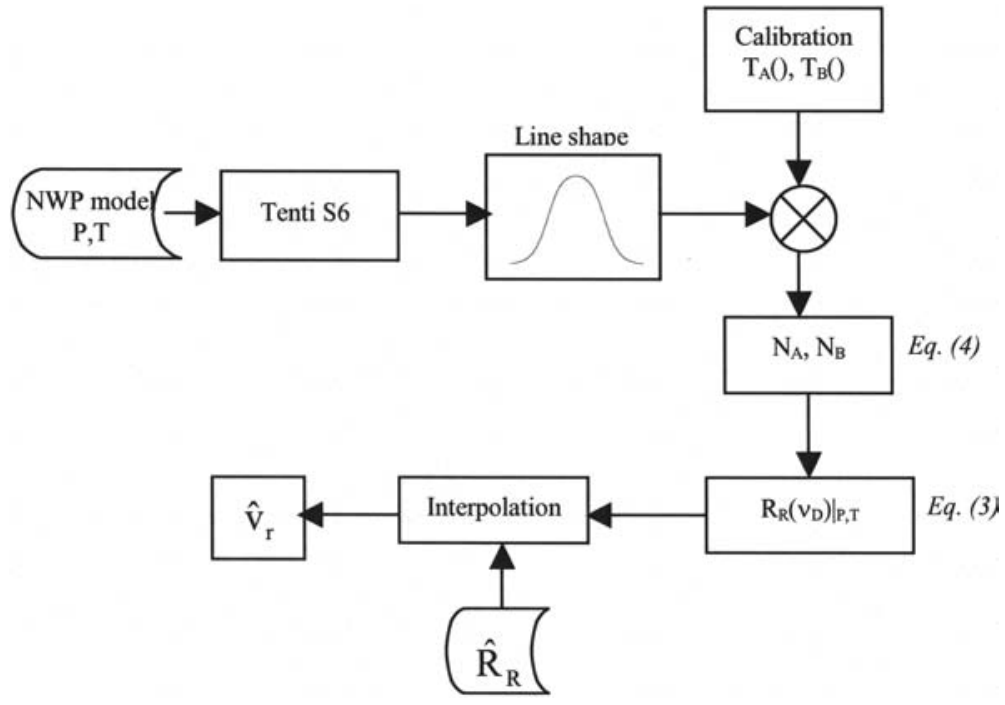

Fig. 9. Diagram showing how a measured Rayleigh response $\hat{R}_{\mathrm{R}}$ can be inverted into a Doppler shift of Rayleigh LOS wind measurement. 
off-line and stored in an auxiliary file (the so-called RayleighBrillouin auxiliary file). It contains the frequency Doppler shifts for large arrays of Rayleigh responses $R(k)$, temperatures $T(j)$ and pressures $P(i)$, which can be summarized by the equation

$\left.R_{\mathrm{R}}[\Phi(i, j, k)]\right|_{P(i), T(j)}=R(k)$.

Numerical tests lead us to retain the following response, pressure and temperature arrays:

$R(k)=-0.5+k \times 0.01, \quad k=0, \ldots, 100$
$T(j)=150 \mathrm{~K}+j \times 1 \mathrm{~K} \quad j=0, \ldots, 200$
$P(i)=10 \mathrm{hPa}+i \times 10 \mathrm{hPa} \quad i=0, \ldots, 103$.

The computation of the 3-D matrix $\Phi(i, j, k)$ involves the computation of the photocounts $N_{\mathrm{A}}$ and $N_{\mathrm{B}}$ for the many pressure and temperature conditions $[P(i), T(j)]$ and a large array of frequency Doppler shifts $v_{d}(m)=-0.5 \times \mathrm{USR}+m \times \mathrm{d} f$, where $m=0, \ldots,(\mathrm{USR} / \mathrm{d} f=60)$ with $\mathrm{USR}=1500 \mathrm{MHz}$ the Useful Spectral Range of the lidar. These numbers are also stored in the Rayleigh-Brillouin auxiliary file for use by the Mie contamination correction.

The total number of elements to be stored is thus $101 \times 104$ $\times 201+2 \times 61 \times 104 \times 201=4661592$, that is about 18 Mbytes of storage place (inverted frequencies and photocounts are coded as 4-byte real numbers). The 3-D matrix has to be renewed each time there is a new characterization of the transmission functions $T_{\mathrm{A}}()$ and $T_{\mathrm{B}}()$. At the present, it is not known how many times this will happen during the mission. As the FP interferometers were designed to achieve the best possible stability, no regular FP calibration procedure has been planned. We anticipate it should not happen more than once a week (worst case).

On the basis of the 3-D matrix $\Phi(i, j, k)$, the inversion of an observed Rayleigh response $\hat{R}_{\mathrm{R}}$ does amount to a simple, fast 3-D interpolation. The result is the inverted Doppler shift $v_{d}$ such that $\left.R_{\mathrm{R}}\left(v_{d}\right)\right|_{P, T}=\hat{R}_{\mathrm{R}}$ where $P$ and $T$ are the pressure and temperature inside the sensing volume (provided by the NWP model fields interpolated to the actual geographical and time coordinates of the observation). By repeating the interpolation two times with $(P+1 \mathrm{hPa}, T)$ and $(P, T+1 \mathrm{~K})$, it is very easy to obtain estimates $\hat{\alpha}_{T}$ and $\hat{\alpha}_{P}$ for the first order derivatives

$\alpha_{T}=\left.\frac{\partial v_{\mathrm{d}}}{\partial T}\left(\hat{\mathrm{R}}_{\mathrm{R}}\right)\right|_{P, T} \quad$ and $\quad \alpha_{P}=\left.\frac{\partial v_{\mathrm{d}}}{\partial P}\left(\hat{R}_{\mathrm{R}}\right)\right|_{P, T}$.

These figures can be used by the assimilation system in order to reflect $P$ and $T$ upgrades into $v_{\mathrm{r}}$, and asses the potential correlation between LOS wind and pressure and temperature errors.

A third interpolation is added in order to obtain an estimate $\hat{\alpha}_{\mathrm{R}}$ of the first order derivative

$\alpha_{\mathrm{R}}=\left.\frac{\partial v_{\mathrm{d}}}{\partial R}\left(\hat{R}_{\mathrm{R}}\right)\right|_{P, T}$

of the inverted frequency This parameter is used in the next section for correcting Mie contamination.

\section{Correction for Mie contamination}

Due to the minor impact of Mie contamination, its correction can be approximated to a first order and treated as an (optional) addition to the basic pressure-temperature correction presented in the previous section. The principle is explained in Fig. 10. The pressure-temperature correction presented in the previous section provides a zeroth order approximation $v_{d}^{0}$ of the inverted Rayleigh response $\hat{R}_{\mathrm{R}}$. As no Mie contamination is assumed at this stage, $v_{d}^{0}$ is different from the 'correct' inversion $v_{d}$, but, following the conclusions of Section 3.4 on the weakness of Mie impact on Rayleigh winds, the difference should be rather small. Therefore, the response increment brought by the Mie return at the true, unknown frequency $v_{d}$ can be approximated by a first order expansion of eq. (3) with respect to the scattering ratio $\rho$

$$
\Delta R_{\mathrm{R}}=\left.(\rho-1) \frac{\partial R_{\mathrm{R}}}{\partial \rho}\left(\hat{R}_{\mathrm{R}}\right)\right|_{P, T}
$$

Fig. 10. Diagram showing how the impact of Mie contamination is corrected. First, the observed Rayleigh response $\hat{R}_{\mathrm{R}}$ is inverted as if there was no Mie contamination by using the blue, response curve. A zeroth order Doppler shift $v_{d}^{0}$ is thus estimated. Then, the increment $\Delta R_{\mathrm{R}}$ that the Mie contamination would add to $\hat{R}_{\mathrm{R}}$ for the prevailing $P$ and $T$ conditions and the Doppler shift $v_{d}^{0}$ is approximated by a first order expansion. This increment is tangentally back-propagated, the slope of the tangent being itself approximated by the first order derivative $\partial R_{\mathrm{R}} / \partial v_{d}\left(v_{d}^{0}\right)$.

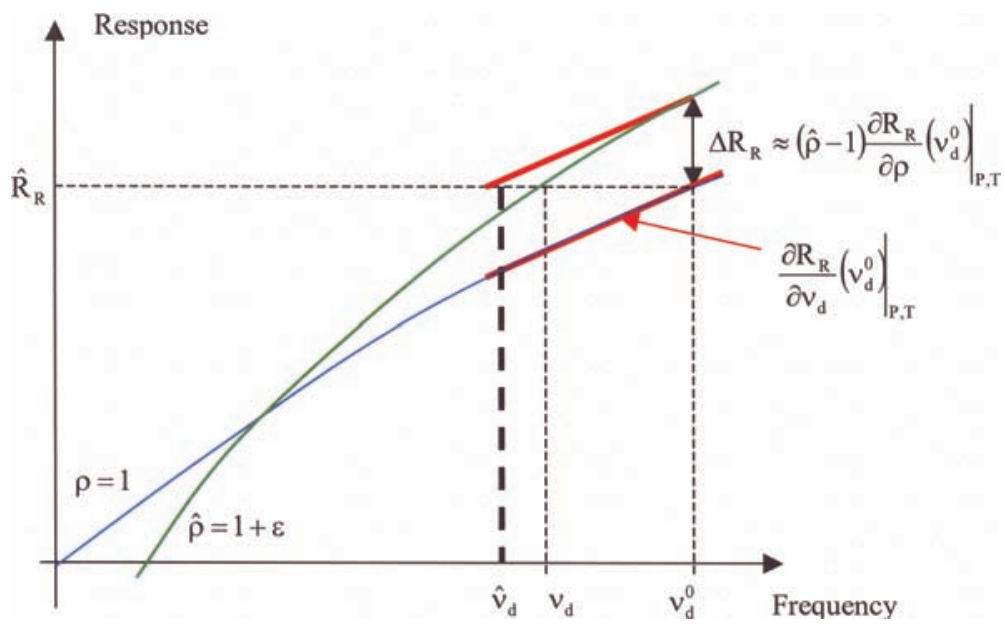


Simple maths show that

$\partial_{\rho} R_{\mathrm{R}}=2 \frac{\partial_{\rho} N_{\mathrm{A}} \times N_{\mathrm{B}}-N_{\mathrm{A}} \times \partial_{\rho} N_{\mathrm{B}}}{\left(N_{\mathrm{A}}+N_{\mathrm{B}}\right)^{2}}$,

where we used the notation $\partial_{\rho} \equiv \partial / \partial \rho . N_{\mathrm{A}}$ and $N_{\mathrm{B}}$ are given by eq. (4). From this equation and eq. (5), it comes

$\partial_{\rho} N_{\mathrm{A}, \mathrm{B}}=K \underbrace{\int T_{\mathrm{A}, \mathrm{B}}(v) I_{\text {mie }}\left(v-v_{d}^{0}\right) \mathrm{d} v}_{\approx T_{\mathrm{A}, \mathrm{B}}\left(v_{d}^{0}\right)}$

The approximation under the horizontal curly brace on the righthand side term is valid because, as discussed in the introduction, the spectrum of the Mie return has a width (FWHM of the order of $75 \mathrm{MHz}$ ) negligible compared to the width of the transmission curves $T_{\mathrm{A}, \mathrm{B}}($ ) (FWHM of $1600 \mathrm{MHz}$ ). Equation (12) thus simplifies to

$\partial_{\rho} R_{\mathrm{R}} \approx 2 \frac{\left.T_{\mathrm{A}}\left(v_{d}^{0}\right) N_{\mathrm{B}}\left(v_{d}^{0}\right)\right|_{P, T, \rho=1}-\left.T_{\mathrm{B}}\left(v_{d}^{0}\right) N_{\mathrm{A}}\left(v_{d}^{0}\right)\right|_{P, T, \rho=1}}{\left[\left.N_{\mathrm{A}}\left(v_{d}^{0}\right)\right|_{P, T, \rho=1}+\left.N_{\mathrm{B}}\left(v_{d}^{0}\right)\right|_{P, T, \rho=1}\right]^{2}}$

which can be easily evaluated by interpolating $T_{\mathrm{A}}$ and $T_{\mathrm{B}}$ to the frequency $v_{d}^{0}$ and the photocounts $\left.N_{\mathrm{A}, \mathrm{B}}\left[v_{d}(m)\right]\right|_{P(i), T(j)}$ stored in Rayleigh-Brillouin auxiliary file to $\left(v_{d}^{0}, P, T\right)$.

The response increment $\Delta R_{\mathrm{R}}$ of eq. (12) is back-propagated by the tangent equation

$\hat{v}_{d}=v_{d}^{0}-\frac{1}{\hat{\alpha}_{\mathrm{R}}} \Delta R_{\mathrm{R}}$,

where the slope of the response curve $\left.R_{\mathrm{R}}\left(v_{d}\right)\right|_{P, T, \rho}$ is approximated by $\hat{\alpha}_{\mathrm{R}}^{-1}$, that is, the estimate of the slope of $\left.R_{\mathrm{R}}\left(v_{d}\right)\right|_{P, T, \rho=1}$.

\section{Conclusions}

The study conducted for the European Space Agency assessed the impact of pressure, temperature and Mie contamination on the retrieval of winds from the signals of the dual-FP receiver of the Rayleigh channel of ADM. It was found that the potential errors of pressure and temperature are above the mission specifications. Due to the sensitivity, the correction of these effects requires that the temperature conditions actually prevailing in the sensing volumes are known with an accuracy of 1 or $2 \mathrm{~K}$ for the temperature. Such a requirement rules out the use of climatology profiles but are in line with the prediction skills of current NWP systems. For the pressure, the requirement is rather coarse, well within the reach of NWP models that achieve pressure accuracies of a few $\mathrm{hPa}$.

The pressure and temperature correction scheme is hosted at the NWP centres as part of the assimilation process of ADM data where first-guess pressure and temperature fields can be space-interpolated to the actual coordinates of ADM observations. It is based on a look-up table approach, that is, interpola- tions through a pre-calculated 3-D matrix containing the inverse frequency shifts for large arrays of pressure and temperature conditions and Rayleigh responses spanning the whole domain of situations likely to be encountered in the atmosphere. The 3-D matrix is based on the transmission curves of the two FP that are characterized by a dedicated calibration procedure, and a pressure and temperature dependant model for the spectrum of the light backscattered by molecules (the Tenti S6 model). Due to expected stability of the FPs, its updating should not happen more than once in a week or once in a month throughout whole lifetime of the mission.

As far as Mie contamination is concerned, its potential biasing impact on Rayleigh winds was found to be small except for cloud returns or aerosol layers loaded unusually heavily. A possible correction scheme was devised that comes as an optional addition to the Rayleigh-Brillouin correction and is based on some of its outputs. Its application depends on the actual availability of some information of the aerosol to molecule backscatter ratio. It may be derived from the Mie channel of the instrument on the condition that this channel probes the same atmospheric volume as the Rayleigh channel. This condition is not met at high altitudes with the nominal instrument settings (the maximum altitude covered by the Mie receiver is well below the maximum altitude sensed by the Rayleigh receiver). An algorithm which does not require Mie signals is currently under development in order to flag out Rayleigh observations with large Mie contaminations. It should be included in the operational version of the level 2B processor of ADM where Mie decontamination is implemented.

The Rayleigh-Brillouin correction scheme presented in this article will generate correlations between pressure and temperature errors in first-guess fields and corrected Rayleigh winds. However, it also provides estimates for the correlation coefficients so that the correlation can be taken into account throughout the assimilation process.

\section{Acknowledgments}

The material contained in the present article results from a study conducted for the European Space Agency under the contract number 1833404/NL/MM. The title of the study was: 'Aeolus: Impact of Line Shape on Wind Measurements and Correction methods'. It was led by P. Flamant (co-author of the present article). We thank all the people at ESTEC who monitored the study and reviewed the final report (M. Endemann, O. Lerille). Our work does also owe much to Pr. L. Létamandia (University of Bordeaux), France, Dr. G. Tenti, and Pr J. P. Boon (Free University of Bruxelles, Belgium), for helpful discussions and clarifications on various Rayleigh-Brillouin theories. The initial code for the Tenti S6 model was obtained from Dr. R. G. Seasholtz from NASA/GRC. 


\section{References}

Abreu, V. J., Barnes, J. E. and Hayes, P. B. 1992. Observation of winds with an incoherent lidar detector. Appl. Opt. 31, 45094514.

Bhatnagar, P. L., Gross, E. P. and Krook, M. 1954. A model for collision processes in Gases. I. Small amplitude processes in charged and neutral one-component system. Phys. Rev. 94, 511-525.

Boley, C. D., Tenti, G. and Desai, R. C. 1972. Kinetic models and Brillouin scattering in a molecular gas. Can. J. Phys. 21582173.

Chanin, M. L., Garnier, A., Hauchecorne, A. and Porteneuve, J. 1989. A Doppler lidar for measuring winds in the middle atmosphere. Geophys. Res. Lett. 16, 1273-1276.

Clark, N. A. 1975. Inelastic light scattering from density fluctuations in dilute gases. The kinetic-hydrodynamic transition in a monatomic gas. Phys. Rev. A 12, 232-244.

Flamant, P., Loth, C., Dabas, A., Denneulin, M.-L., Dolfi-Bouteyre, A. and co-authors. 2005. ILIAD: Impact of Line Shape on Wind Measurements and Correction Methods. Final report ESTEC contract 1833404/NL/MM. 124 pp.

Garnier, A. and Chanin, M. L. 1992. Description of a Doppler Rayleigh lidar for measuring winds in the middle atmosphere. Appl. Phys. B55, $35-40$.

Killeen, T. L., Kennnedy, B. C., Hays, P. B., Symanow, D. A. and Ceckowski, D. H. 1983. Image plane detector for the dynamics explorer Fabry-Perot interferometers. Appl. Opt. 22, 3503-3513.

McGill, M. J., Skinner, W. R. and Irgang, T. D. 1997. Analysis techniques for the recovery of winds and backscatter coefficients from a multiple-channel incoherent Doppler lidar. Appl. Opt. 36, 12531268.
Pan, X., Shneider, M. N. and Miles, R. B. 2002a. Coherent Rayleigh-Brillouin scattering in monatomic gases in the kinetic regime. Paper \#AIAA-2002-3235, 22nd AIAA Aerodynamic Measurement Technology and Ground Testing Conference, St. Louis, MO, June 24-26.

Pan, X., Shneider, M. N. and Miles, R. B. 2002b. Coherent RayleighBrillouin scattering. Phys. Rev. Lett. 89, article \#183001.

Pan, X. G., Shneider, M. N. and Miles, R. B. 2004. Coherent RayleighBrillouin scattering in molecular gases. Phys. Rev. A 69, Article \#033814.

Rye, B. J. 1998. Molecular backscatter heterodyne lidar: a computational evaluation. Appl. Opt. 37, 6321-6328.

Souprayen, C., Garnier, A., Hertzog, A., Hauchecorne, A. and Porteneuve, J. 1999a. Rayleigh-Mie Doppler wind lidar for atmospheric measurements. I. Instrumental setup, validation, and first climatological results. Appl. Opt. 38, 2410-2421.

Souprayen, C., Garnier, A. and Hertzog, A. 1999b. Rayleigh-Mie Doppler wind lidar for atmospheric measurements. II. Mie scattering effect, theory, and calibration. Appl. Opt. 38, 2422-2431.

Tan, D. G. H., Andersson, E., De Kloe, J., Marseille, G.-J., Stoffelen, A. and co-authors. 2007. The ADM-Aeolus wind retrieval algorithms. Tellus 60A, doi:10.1111/j.1600-0870.2007.00285.x.

Tenti, G., Boley, C. D. and Desai, R. C. 1974. On the kinetic model description of Rayleigh-Brillouin scattering from molecular gases. Can. J. Phys. 52, 285.

Wegdam, G. H. and Schaink, H. M. 1989. Light-scattering studies of dynamical processes in disparate mass gas mixtures. Phys. Rev. A $\mathbf{4 0}$, 7301-7311.

Yip, S. and Nelkin, M. 1964. Application of kinetic model to timedependent density correlations in fluids. Phys. Rev. 135, A1241A1247. 